\title{
Triple-negative breast cancer: BRCAness and concordance of clinical features with BRCA1-mutation carriers
}

\author{
E H Lips ${ }^{1,2}$, L Mulder ${ }^{1,2}$, A Oonk ${ }^{3}$, L E van der Kolk ${ }^{4}$, F B L Hogervorst ${ }^{2}$, A L T Imholz ${ }^{3}$, J Wesseling ${ }^{1,2}$, \\ S Rodenhuis ${ }^{5}$ and P M Nederlof ${ }^{\star}, 2$ \\ ${ }^{1}$ Department of Molecular Pathology, The Netherlands Cancer Institute, Plesmanlaan 121, 1066 CX Amsterdam, The Netherlands; \\ ${ }^{2}$ Department of Pathology, The Netherlands Cancer Institute, Plesmanlaan 121, 1066 CX Amsterdam, The Netherlands; \\ ${ }^{3}$ Department of Internal Medicine, Deventer Hospital, Nico Bolkesteinlaan 75, 7416 SE Deventer, The Netherlands; ${ }^{4}$ Department \\ of Clinical genetics, The Netherlands Cancer Institute, Plesmanlaan 121, 1066 CX Amsterdam, The Netherlands and ${ }^{5}$ Department \\ of Medical Oncology, The Netherlands Cancer Institute, Plesmanlaan 121, 1066 CX Amsterdam, The Netherlands
}

Background: BRCAness is defined as shared tumour characteristics between sporadic and BRCA-mutated cancers. However, how to exactly measure BRCAness and its frequency in breast cancer is not known. Assays to establish BRCAness would be extremely valuable for the clinical management of these tumours. We assessed BRCAness characteristics frequencies in a large cohort of triple-negative breast cancers (TNBCs).

Methods: As a measure of BRCAness, we determined a specific BRCA1-like pattern by array Comparative Genomic Hybridisation $(\mathrm{aCGH})$, and BRCA1 promoter methylation in 377 TNBCs, obtained from 3 different patient cohorts. Clinicopathological data were available for all tumours, BRCA1-germline mutation status and chemotherapy response data were available for a subset.

Results: Of the tumours, $66-69 \%$ had a BRCA1-like aCGH profile and $27-37 \%$ showed BRCA1 promoter methylation. BRCA1germline mutations and BRCA1 promoter methylation were mutually exclusive events $\left(P=1 \times 10^{-5}\right)$. BRCAness was associated with younger age and grade 3 tumours. Chemotherapy response was significantly higher in BRCA1-mutated tumours, but not in tumours with BRCAness (63\% (12 out of 19) vs 35\% (18 out of 52) pathological complete remission rate, respectively).

Conclusion: The majority of the TNBCs show BRCAness, and those tumours share clinicopathological characteristics with BRCA1-mutated tumours. A better characterisation of TNBC and the presence of BRCAness could have consequences for both hereditary breast cancer screening and the treatment of these tumours.

The triple-negative breast cancer (TNBC) subtype occurs in $15-20 \%$ of cases and is associated with rapid growth, early metastasis and a worse prognosis than other breast cancer subtypes (Bauer et al, 2007; Blows et al, 2010). Among breast cancer patients with a hereditary BRCA1 mutation, over $80 \%$ is a TNBC (Lakhani et al, 2002). As sporadic triple-negative tumours often show the same histological characteristics and clinical outcome as BRCA1mutation carriers, several studies postulated that BRCA1 inactivation might also have a role in sporadic TNBCs (Turner et al, 2007;
Joosse et al, 2009, 2011; Silver et al, 2010). The phenotype that some sporadic tumours share traits with familial-BRCA cancer is called BRCAness and is described in a landmark paper by Turner et al (2004). The importance of defining such a group of tumours lies within the clinical management of these tumours. As BRCA1 and BRCA2 are involved in the repair of DNA double-strand breaks (DSBs), a process called homologous recombination, dysfunctional BRCA proteins could make a tumour extra sensitive for drugs inducing those DNA DSBs. Indeed, it has been shown 
that BRCA1- and BRCA2-mutation carriers have a high sensitivity to alkylators or the new class of PARP inhibitors (Fong et al, 2009; Kriege et al, 2009), and not to spindle poisons, such as taxanes (Kriege et al, 2012).

We have found that breast tumours from BRCA-mutation carriers show specific array Comparative Genomic Hybridisation $(\mathrm{aCGH})$ profiles and that these tumour profiles are also present in some sporadic breast cancer patients (Joosse et al, 2009, 2012). As such, we assume that they represent BRCAness, and that BRCAlike profiles are a measure of BRCAness. The aCGH BRCA1-like tumour profile is characterised by genomic instability in general, but also by specific alterations, such as gain of $3 \mathrm{q}$, loss of $5 \mathrm{q}$, and more. A large proportion of these BRCA1-like tumours have either a BRCA1 mutation or show somatic BRCA1 promoter methylation (Lips et al, 2011b). Possibly, a currently unknown genetic alteration in the BRCA1 pathway or a related event could explain the remaining BRCA1-like cases. The BRCA2-like aCGH profile is characterised by even more genomic aberrations. The clinical use of these genomic profiles is twofold. In genetic counselling, an aCGH BRCA1 or BRCA2-like genomic profile is an extra indication of BRCA1/2 involvement, whereas its absence can help to rule out a hereditary component (Joosse et al, 2009). Also, it can help to classify variants of unknown significance (VUS). The genomic profiles can also be used to select patients for specific chemotherapy regimens. We previously showed that tumours with an aCGH BRCA1-like profile respond favourably to intensified alkylating chemotherapy (Vollebergh et al, 2011).

What the best method is to measure BRCAness is still not known. Therefore, also the extent of BRCAness in TNBC is currently unknown. Previously, we showed that BRCA1-related abnormalities (aCGH BRCA1-like profile, BRCA1 promoter methylation and low BRCA1 mRNA expression) were predominantly observed in TN tumours, whereas a BRCA2-like profile was mainly observed in ER + tumours (Lips et al, 2011b). Therefore, we determined aCGH BRCA1-like profiles and BRCA1 promoter methylation, as indicators of BRCAness, in a large cohort of TNBCs. In addition, we assessed the association with clinicopathological variables and treatment response, and compared the BRCAness patients with BRCA1-mutation carriers.

\section{MATERIALS AND METHODS}

Patients. The first series of TNBC samples (the 'neoadjuvant series') consists of pre-treatment biopsies of primary breast tumours from 152 women with TNBC. All patients received neoadjuvant treatment at the Netherlands Cancer Institute between 2004 and 2012 as part of ongoing clinical trials (Rodenhuis et al, 2010; Lips et al, 2011b). Most patients received six courses of cyclophosphamide and doxorubicin, administered in a dose-dense schedule (ddAC) (every 2 weeks). After the last course of chemotherapy, all patients underwent breast-conserving therapy or mastectomy according to the standard protocols at our institute.

The second series of samples (the 'familial series') consists of tumours of patients suspected to have a hereditary form of breast cancer who were referred for further molecular diagnostic testing, usually after a negative BRCA1 mutation screening result or if germline DNA for mutation screening was not available, but BRCAness was suspected.

The third sample series (the 'adjuvant series') consists of tumours of patients who received adjuvant chemotherapy at the Deventer hospital (Oonk et al, 2012).

Clinicopathological variables were obtained from patient records. The three different series came from studies which had been approved by the institutional review board and informed consent was obtained from all patients.
Pathology. Triple-negative status was defined by the absence of ER and PR expression and no amplification of HER2. More than $10 \%$ positive immunohistochemistry staining was used as a cutoff to call a sample negative. HER2 staining was either 0 or $1+$. In case of a $2+$ staining, chromogenic in situ hybridisation was performed to determine HER2 amplification (gene copy number $\geqslant 6$ per tumour cell). Chemotherapy response was assessed in the surgery resection specimen. The complete absence of any invasive tumour tissue in the breast and the lymph nodes was considered as a pathological complete response (pCR). All other responses were grouped in the no-pCR group. All pathology slides were reviewed by an experienced breast cancer pathologist (JW).

BRCA1-mutation analysis. BRCA1-mutation status was obtained from patient records, obtained through our family cancer clinic. Briefly, germline DNA was isolated from peripheral blood lymphocytes of affected patients. We used mutation scanning methods. The Protein Truncation test was used for exon 11 of BRCA1 and exons 10 and 11 or BRCA2. The remaining exons were tested using Denaturing Gradient Gel Electrophoresis (DGGE). Confirmation of aberrant samples was done by Sanger sequencing (van der Hout et al, 2006). In addition, Multiplex Ligation-dependent Probe Amplification (MLPA) was performed using MLPA kit P087 (BRCA1; MRC-Holland, Amsterdam, The Netherlands) to detect large genomic deletions or duplications in the genes. For patients who did not visit the family cancer clinic, no mutation analysis could be performed, as those patients did not give informed consent for this specific analysis.

aCGH BRCA1-like genomic profile. aCGH BRCA1-like scores were obtained by three different assays. The older samples (2004-2010) were obtained by a 3.5k BAC array CGH platform. The DNA segments covered the whole genome with an average spacing of $1 \mathrm{MB}$. The protocols have been described before (Joosse et al, 2007). Classification of subtypes was performed using the aCGH BRCA1-like classifier developed by Joosse et al (2009). The cutoff for a BRCA1-like aCGH pattern was 0.5 . The more recent samples were analysed using Nimblegen 128K oligo arrays or Multiplex Ligation Probe Amplification (MLPA) assay (P376 BRCA1ness; MRC-Holland, Amsterdam, The Netherlands) (Lips et al, 2011a). For the Nimblegen arrays labelling, hybridisation, imaging and data analysis have been performed by manufacturer's protocol (Nimblegen, Roche, Madison, WI, USA). The translation and concordance between the BAC aCGH BRCA1-like classifier and the Nimblegen classifier have been described recently (Philip Schouten, personal communication, March 2013). The BRCAlness MLPA assay was performed according to manufacturer's guidelines. Analysis procedure and validation with the aCGH has been described previously (Lips et al, 2011a).

BRCA1 promoter methylation. Hypermethylation of the BRCA1 promoter was determined using Methylation-specific MLPA analysis, according to manufacturers' protocol. For the neoadjuvant series the ME005-custom assay was performed, while the familial-breast cancer series was typed with ME-001 (both from MRC-Holland). For normalisation and analysis, the Coffalyzer program was used (MRC-Holland). We used a cutoff of $20 \%$ to call a sample methylated. This cutoff was according to manufacturer's protocol (www.mlpa.com). Employing this cutoff, methylated samples show a very low BRCA1 gene expression (Joosse et al, 2011; Lips et al, 2011b).

Statistical tests. The Fisher's exact test was used to assess association between the dichotomised histological and clinical variables and BRCAness characteristics. Logistic regression was performed to adjust for the following variables: age, T-stage, $\mathrm{N}$-stage, and grade. Data analysis was performed using IBM SPSS Statistics version 20 (IBM, Chicago, IL, USA). 


\section{RESULTS}

In this study, we combined data from three different patient cohorts. As these are retrospective series not all variables are available for every tumour. Table 1 shows patient characteristics for each series. Patients in the neoadjuvant and in the familial series were younger $(P<0.001)$ and had tumours with a lower stage $(P<0.001)$, but more often positive lymph nodes $(P=0.01)$ than tumours in the Adjuvant series. In all series, the percentage grade 3 tumours was high, although in the neoadjuvant series a substantial part $(30 \%)$ had grade 1 or 2 tumours $(P<0.001)$. Grading in this series was assessed on biopsy material. As breast tumours are often heterogeneous, it could be that only the lowgrade part is present in the biopsy. This can lead to an underestimation of grade in this sample series.

In the neoadjuvant series, germline BRCA1-mutation analysis was performed for 112 patients, 27 showed a mutation (24\%) (Table 2a). In the familial-breast cancer series, no mutations were present, as those tumours were referred for additional molecular testing after a negative BRCA1 mutation test result. However, a substantial percentage has an unclassified variant for BRCA1 $(22 \%)$. For the Adjuvant series we only know which patients have a BRCA1- or BRCA2-germline mutation, not which patients were actually tested, making it unreliable to give percentages.

In the TNBCs without a germline BRCA1 mutation, we determined the percentage of tumours with a BRCA1-like aCGH profile and with BRCA1 promoter methylation (Table $2 \mathrm{~b}$ ). The percentage BRCA1-like aCGH tumours was similar in the three series, it ranged from $66 \%$ to 69 of all patients tested. BRCA1 promoter methylation was slightly more frequent in the familialbreast cancer series, $37 \%$ of the cases showed promoter methylation, while it was $29 \%$ in the neoadjuvant series and $27 \%$ in the adjuvant series. These percentages were not significantly different.

To assess the co-occurrence of the two BRCAness measures and BRCA1 mutation, we analysed the neoadjuvant series, as this series was the most complete for the three data types and not biased towards familial cases. For 88 cases, all data were complete, meaning that we had BRCA1/2-germline mutation status, aCGH and a methylation assay performed. In this set, we established the overlap and association between the different data types. In all, $76 \%$ of the TNBC cases had a BRCA1-like aCGH profile (Figure 1). Of these, aCGH BRCA1 likes, $30 \%$ had BRCA1 promoter methylation, while $16 \%$ had a germline BRCA1 mutation. For the remaining aCGH BRCA1 likes, we do not have yet an explanation of the BRCAness phenotype and indicated them by 'Other', meaning that another mechanism is responsible for the BRCA1-like aCGH genomic profile. There are 3 (4\%) tumours with a mutation and $3(4 \%)$ with BRCA1 promoter methylation that do not show a BRCA1-like aCGH profile. Revision of these samples by a pathologist showed that they all had lymphatic infiltrate or too many normal cells in the biopsy material, which diminishes the tumour aCGH profile. We should note that it is difficult to exactly estimate the amount of infiltrate in a biopsy. When the tumour cell percentage is well below $50 \%$, the tumour profile will flatten towards that of a normal-normal hybridisation ('flatliner'). Such an aCGH profile without any aberrations is highly unlikely for TNBCs, which show in general a high level of

\begin{tabular}{|c|c|c|c|c|c|c|c|c|}
\hline \multicolumn{5}{|c|}{ Table 1. Patient characteristics } & \multicolumn{4}{|c|}{ Table 2. BRCA mutations and BRCAness frequencies } \\
\hline & Neoadjuvant & Familial & Adjuvant & R. & \multicolumn{4}{|c|}{ a. Frequency of BRCA mutations in all TNBCs ${ }^{a}$} \\
\hline $\begin{array}{l}\text { Age, median } \\
\text { (range) }\end{array}$ & 42 (19-72) & $39(18-67)$ & $50(23-75)$ & $<0.001$ & & $\begin{array}{l}\text { Neoadjuvant } \\
\quad(n=152)\end{array}$ & $\begin{array}{l}\text { Familial } \\
(n=134)\end{array}$ & $\begin{array}{l}\text { Adjuvant } \\
(n=91)\end{array}$ \\
\hline \multicolumn{5}{|l|}{ Grade } & \multicolumn{4}{|c|}{ Mutation analysis } \\
\hline $\begin{array}{l}\text { Grade } 1 / 2 \\
\text { (\%) }\end{array}$ & $42(30)$ & $11(11)$ & $4(5)$ & $<0.001$ & \multirow{4}{*}{$\begin{array}{l}\text { BRCA1 (\%) } \\
\text { BRCA2 (\%) } \\
\text { BRCA1 UV (\%) } \\
\text { BRCA2 UV (\%) } \\
\text { Wild type (\%) } \\
\text { ND }\end{array}$} & \multirow{4}{*}{$\begin{aligned} & 27(24) \\
& 2(2) \\
& 2(2) \\
& 1(1) \\
& 80(71) \\
& 40\end{aligned}$} & \multirow{4}{*}{$\begin{array}{c}0(0) \\
0(0) \\
16(22) \\
7(9) \\
51(69) \\
60\end{array}$} & \multirow{4}{*}{$\begin{array}{r}9 \\
4 \\
0 \\
0 \\
0 \\
78\end{array}$} \\
\hline Grade 3 (\%) & $98(70)$ & $87(89)$ & $69(95)$ & & & & & \\
\hline ND & 12 & 43 & 18 & & & & & \\
\hline \multirow{2}{*}{\multicolumn{5}{|c|}{ Histology }} & & & & \\
\hline & & & & & \multicolumn{4}{|c|}{ b. Frequency of BRCAness in all TNBCs without BRCA1/2 mutations ${ }^{b}$} \\
\hline IDC (\%) & 114 (95) & $115(92)$ & $0(0)$ & 0.99 & & \multirow{2}{*}{$\begin{array}{c}\text { Neoadjuvant } \\
(n=123)\end{array}$} & \multirow{2}{*}{$\begin{array}{l}\text { Familial } \\
(n=134)\end{array}$} & \multirow{2}{*}{$\begin{array}{l}\text { Adjuvant } \\
(n=78)\end{array}$} \\
\hline ILC (\%) & $2(2)$ & $2(2)$ & $0(0)$ & & & & & \\
\hline other (\%) & $4(3)$ & $7(6)$ & $0(0)$ & & \multirow{2}{*}{\multicolumn{4}{|c|}{$\mathrm{aCGH}$}} \\
\hline ND & 32 & 17 & 91 & & & & & \\
\hline \multicolumn{5}{|l|}{ T-stage } & \multirow{2}{*}{$\begin{array}{l}\text { BRCA1 like (\%) } \\
\text { Non-BRCA1 } \\
\text { like (\%) } \\
\text { ND }\end{array}$} & \multirow{2}{*}{$\begin{array}{l}70(66) \\
35(34) \\
18\end{array}$} & \multirow{2}{*}{$\begin{array}{l}92(69) \\
42(31) \\
0\end{array}$} & \multirow{2}{*}{$\begin{array}{l}53(68) \\
25(32) \\
0\end{array}$} \\
\hline T1/2 (\%) & $96(75)$ & $38(90)$ & $7(8)$ & $<0.001$ & & & & \\
\hline T3/4 (\%) & $32(25)$ & $4(10)$ & $84(92)$ & & \multirow{2}{*}{\multicolumn{4}{|c|}{ BRCA1 promoter methylation }} \\
\hline ND & 24 & 99 & 0 & & & & & \\
\hline \multirow{2}{*}{\multicolumn{5}{|c|}{ Nodal stage }} & \multirow{3}{*}{$\begin{array}{l}\text { Methylated (\%) } \\
\text { Unmethylated (\%) } \\
\text { ND }\end{array}$} & \multirow{3}{*}{$\begin{array}{l}31(29) \\
75(71) \\
17\end{array}$} & \multirow{3}{*}{$\begin{array}{l}47(37) \\
81(63) \\
6\end{array}$} & \multirow{3}{*}{$\begin{array}{l}21(27) \\
56(73) \\
1\end{array}$} \\
\hline & & & & & & & & \\
\hline Negative (\%) & $47(36)$ & $24(57)$ & $49(54)$ & 0.01 & & & & \\
\hline Positive (\%) & $82(64)$ & $18(43)$ & $42(46)$ & & \multirow{3}{*}{\multicolumn{4}{|c|}{ 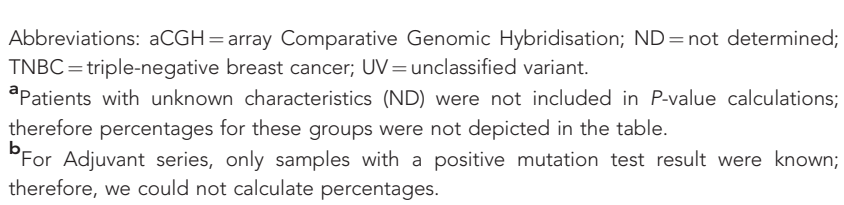 }} \\
\hline ND & 23 & 99 & 0 & & & & & \\
\hline \multicolumn{5}{|c|}{$\begin{array}{l}\text { Abbreviations: IDC = invasive ductal carcinoma; ILC = invasive lobular carcinoma; ND= not } \\
\text { determined. Patients with unknown characteristics (ND) were not included in P-value } \\
\text { calculations; therefore, percentages for these groups were not depicted in the table. }\end{array}$} & & & & \\
\hline
\end{tabular}




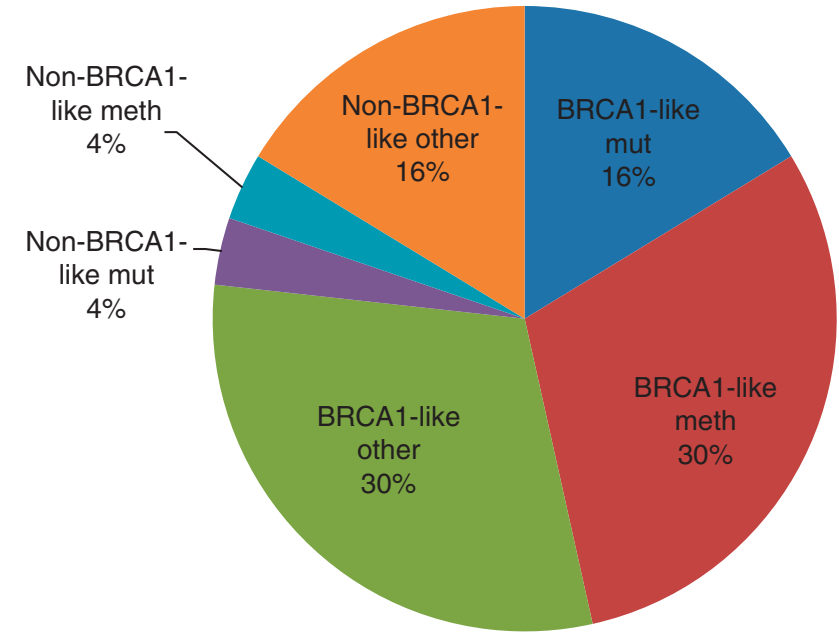

Figure 1. Co-occurrence of aCGH BRCA1-like profile, BRCA1 mutation, and BRCA1 methylation. This pie chart shows the frequency of the combination of BRCAness characteristics and BRCA1 mutation status in 88 tumours from the neoadjuvant series. aCGH BRCA1-like tumours were either mutated (Mut), methylated (Meth) or showed no aberration (Other). The same applied to the non-BRCA1-like tumours.

\begin{tabular}{|c|c|c|c|}
\hline & $\begin{array}{l}\text { BRCA1 } \\
\text { mutation }\end{array}$ & $\begin{array}{c}\text { No BRCA1 } \\
\text { mutation }\end{array}$ & Total \\
\hline Methylated & 0 & 52 & 52 \\
\hline Unmethylated & 31 & 97 & 128 \\
\hline Total & 31 & 149 & 180 \\
\hline
\end{tabular}

genomic instability. Therefore, we consider these six samples as false negatives for a BRCA-like aCGH profile.

Interestingly, no cases were found in this study with a concurrent BRCA1-germline mutation and somatic BRCA1 promoter methylation (Table 3). For 180 samples, both data types were available. In all, 52 out of 149 non-BRCA1-mutated cancers (35\%) showed BRCA1 promoter methylation, while there were not any mutation carriers with methylation ( 0 out of 31 BRCA1mutated tumours $)\left(P=1 \times 10^{-5}\right.$, Fisher's Exact test). If BRCA1 promoter methylation analysis would be performed to exclude a germline BRCA1 mutation, then the specificity would be $100 \%$ (31 out of 31 ). Positive predictive value would be $100 \%$ (52 out of $52)$ as well. Sensitivity and negative predictive values are $35 \%$ (52 out of 149 ) and $24 \%$ (31 out of 128), respectively.

We determined the association between clinical variables and BRCA1 mutation, BRCA1-like aCGH, and BRCA1 promoter methylation status. BRCA1-like aCGH and BRCA1 promoter methylated tumours were diagnosed at a younger age than nonBRCA1-like aCGH or non-BRCA1-methylated tumours $(P=0.04$ and $P=0.01$, respectively). BRCA1-mutated and BRCA1-like aCGH tumours were more often histological grade 3 tumours (94\% vs $74 \%(P=0.02)$ for BRCA1-mutated $v s$ wild-type tumours and $86 \%$ vs $67 \%(P=0.01)$ for aCGH BRCA1-like versus aCGH non-BRCA1-like tumours) (Table 4$)$. There were minor differences in $\mathrm{T}$ - and $\mathrm{N}$-stage between the groups.

Neoadjuvant chemotherapy response data and recurrence data were available for the neoadjuvant series and recurrence data were available for the Adjuvant series. Patients with a BRCA1 mutation had a higher response rate for neoadjuvant chemotherapy $(63 \%$ (12 out of 19)) than patients without a mutation (33\% (23 out of 69)) $(P=0.02)$. After adjusting for age, T-stage, $\mathrm{N}$-stage, and grade in a multivariate model, there was a trend for a better response in BRCA1-mutated tumours $(P=0.08)$. The patients with a BRCA1-like aCGH profile $(35 \%$ vs $21 \%$, $P=0.22)$ and BRCA1 promoter methylation (46\% vs $31 \%$, $P=0.20)$ showed higher response rates than the aCGH nonBRCA1-like or non-methylated groups. However, these rates were not significantly different. There was no difference in percentage recurrences between groups.

\section{DISCUSSION}

BRCAness is the phenotype that some sporadic tumours share with BRCA-mutated cancers. These tumours can have BRCA1 promoter methylation, a somatic mutation or another alteration causing a dysfunctioning BRCA pathway. We hypothesise that those tumours have a defective DNA DSB repair, as BRCA1 and BRCA2-mutation carriers have. This defect makes them extra sensitive to DNA DSBs that are induced by chemotherapy. In the current study, we determined the frequency of BRCAness in three cohorts of TNBCs. In addition, we assessed if BRCAness was associated with specific clinicopathological variables and with chemotherapy response.

In this study, nearly $70 \%$ of the TNBCs show a BRCA1-like aCGH pattern and $27-37 \%$ show BRCA1 promoter methylation. The reason that the percentage of aCGH BRCA1-like tumours is so high in TNBC may be caused by the approach the aCGH BRCA1like classifier was built. It was developed by comparing BRCA1mutated breast tumours with sporadic tumours. This latter group consists of $30 \%$ TNBCs, while $95 \%$ of the BRCA1-mutated tumours were TNBCs, resulting in a bias for TNBCs in the aCGH BRCA1-like classifier. Therefore, it could be that the TNBCs are just classified as aCGH BRCA1-like due to their triple-negative status. On the other hand, it may be a biological effect; the TNBC phenotype and BRCA1 inactivation are highly correlated and not independent factors. The fact that in the TNBC cases $\sim 20-30 \%$ of the cases are BRCA1-germline mutation carriers and an additional $30-40 \%$ of the cases show BRCA1 inactivation by somatic BRCA1 promoter methylation is a strong argument for the latter. In fact, BRCA1 inactivation by somatic mutation of BRCA1 could possibly explain (part of) the remaining aCGH BRCA1-like cases. This is currently under evaluation. Also, other genes of the DNA damage response pathway, where BRCA1 and BRCA2 have a role, might be involved (Turner et al, 2004). For example, methylation of FANC genes has been described (Turner et al, 2004; Cancer Genome Atlas Network, 2012).

By comparing histopathological variables we found that BRCA1-like aCGH tumours resemble the BRCA1-mutated tumours: they occur in younger patients and are more often grade 3 tumours. In a previous study, we also found that patients with aCGH BRCA1-like tumours were younger, and had less often positive nodes than non-BRCA1 likes (Oonk et al, 2012). A study by Foulkes found that the pattern of metastatic spread of familialBRCA1 cancers seems to be different, with a low incidence of lymphatic spread to the axillary nodes, but a high incidence of haematogenous spread manifesting in a poor prognosis (Foulkes et al, 2003). It could be that aCGH BRCA1-like tumours resemble BRCA1-mutated tumours also in this aspect.

We found a better chemotherapy response to anthracyclinebased chemotherapy in tumours from BRCA1-mutation carriers and slightly higher response percentages in methylated and aCGH BRCA1-like tumours, although not significant. Others found that BRCA1-mutated or methylated tumours were more 
Table 4. Association between BRCA1 mutations and BRCAness characteristics with clinical variables

\begin{tabular}{|c|c|c|c|c|c|c|c|c|c|}
\hline & \multicolumn{3}{|c|}{$\begin{array}{l}\text { Patients screened for BRCA1 } \\
\text { mutations }\end{array}$} & \multicolumn{3}{|c|}{$\begin{array}{l}\text { aCGH BRCA1 like in non-mutation } \\
\text { carriers }\end{array}$} & \multicolumn{3}{|c|}{$\begin{array}{c}\text { Methylation in non-mutation } \\
\text { carriers }\end{array}$} \\
\hline & $\begin{array}{l}\text { No BRCA1 } \\
\text { mutation }\end{array}$ & $\begin{array}{l}\text { BRCA1 } \\
\text { mutation }\end{array}$ & $\boldsymbol{P}$-value & $\begin{array}{c}\text { Non- } \\
\text { BRCA1 like }\end{array}$ & BRCA1 like & $\boldsymbol{P}$-value & $\begin{array}{c}\text { No } \\
\text { methylation }\end{array}$ & Methylated & $P$-value \\
\hline $\begin{array}{l}\text { Age, median } \\
\text { (range) }\end{array}$ & $41(18-67)$ & $37(23-68)$ & 0.26 & $49(18-67)$ & $44(18-75)$ & 0.04 & 47 (18-75) & $40(19-75)$ & 0.01 \\
\hline \multicolumn{10}{|l|}{ Grade } \\
\hline $\begin{array}{l}\text { Grade } 1 / 2(\%) \\
\text { Grade } 3(\%)\end{array}$ & $\begin{array}{l}35(26) \\
102(74)\end{array}$ & $\begin{array}{c}2(6) \\
30(94)\end{array}$ & 0.02 & $\begin{array}{l}25(33) \\
51(67)\end{array}$ & $\begin{array}{l}24(14) \\
151(86)\end{array}$ & 0.01 & $\begin{array}{l}30(18) \\
137(82)\end{array}$ & $\begin{array}{l}16(19) \\
67(81)\end{array}$ & 0.80 \\
\hline \multicolumn{10}{|l|}{ Histology } \\
\hline $\begin{array}{l}\text { IDC (\%) } \\
\text { ILC (\%) }\end{array}$ & $\begin{array}{c}126(98) \\
3(2)\end{array}$ & $\begin{array}{c}20(100) \\
0(0)\end{array}$ & 0.49 & $\begin{array}{l}60(97) \\
2(3)\end{array}$ & $\begin{array}{c}127(99) \\
1(1)\end{array}$ & 0.21 & $\begin{array}{c}121(99) \\
1(1)\end{array}$ & $\begin{array}{l}61(97) \\
2(3)\end{array}$ & 0.23 \\
\hline \multicolumn{10}{|l|}{ T-stage } \\
\hline $\begin{array}{l}\mathrm{T} 1 / 2(\%) \\
\mathrm{T} 3 / 4(\%)\end{array}$ & $\begin{array}{l}83(73) \\
30(27)\end{array}$ & $\begin{array}{l}15(54) \\
13(46)\end{array}$ & 0.04 & $\begin{array}{l}38(55) \\
31(45)\end{array}$ & $\begin{array}{l}73(52) \\
68(48)\end{array}$ & 0.65 & $\begin{array}{l}78(54) \\
67(46)\end{array}$ & $\begin{array}{l}31(50) \\
31(50)\end{array}$ & 0.62 \\
\hline \multicolumn{10}{|l|}{ Nodal stage } \\
\hline $\begin{array}{l}\text { Negative (\%) } \\
\text { Positive (\%) }\end{array}$ & $\begin{array}{l}49(43) \\
64(57)\end{array}$ & $\begin{array}{l}14(48) \\
15(52)\end{array}$ & 0.64 & $\begin{array}{l}23(34) \\
45(66)\end{array}$ & $\begin{array}{l}70(49) \\
72(51)\end{array}$ & 0.04 & $\begin{array}{l}63(43) \\
82(57)\end{array}$ & $\begin{array}{l}30(48) \\
32(52)\end{array}$ & 0.52 \\
\hline \multicolumn{10}{|l|}{ Response } \\
\hline $\begin{array}{l}\text { No pCR (\%) } \\
\text { PCR (\%) }\end{array}$ & $\begin{array}{l}46(67) \\
23(33)\end{array}$ & $\begin{array}{c}7(37) \\
12(63)\end{array}$ & 0.02 & $\begin{array}{l}22(79) \\
6(21)\end{array}$ & $\begin{array}{l}34(65) \\
18(35)\end{array}$ & 0.22 & $\begin{array}{l}40(69) \\
18(31)\end{array}$ & $\begin{array}{l}13(54) \\
11(46)\end{array}$ & 0.20 \\
\hline \multicolumn{10}{|l|}{ Recurrence } \\
\hline $\begin{array}{l}\text { No relapse (\%) } \\
\text { Relapse (\%) }\end{array}$ & $\begin{array}{l}60(80) \\
15(20)\end{array}$ & $\begin{array}{c}25(89) \\
3(11)\end{array}$ & 0.27 & $\begin{array}{l}42(79) \\
11(21)\end{array}$ & $\begin{array}{l}82(77) \\
25(23)\end{array}$ & 0.71 & $\begin{array}{l}86(75) \\
28(25)\end{array}$ & $\begin{array}{l}37(79) \\
10(21)\end{array}$ & 0.66 \\
\hline
\end{tabular}

sensitive to anthracycline- or platinum-based chemotherapy (Kriege et al, 2009; Silver et al, 2010). In a previous study, we found that aCGH BRCA1-like tumours had a higher sensitivity to high-dose platinum-based chemotherapy but not to standard anthracycline-based chemotherapy (Vollebergh et al, 2011). In the current study, patients were treated with a dose dense regimen, which is different from the regimens in the previous study. We are now performing a prospective neoadjuvant study where patients with an aCGH BRCA1-like profile are randomised between standard anthracycline-based chemotherapy and high-dose platinum-based chemotherapy (NCT01057069). Others found in studies with BRCA1-mutation carriers and tumours with decreased BRCA1 gene expression, a high sensitivity to DSB-inducing drugs, such as platinum, but also to the new drug class of PARP inhibitors (Fong et al, 2009; Silver et al, 2010).

Interestingly, there were no tumours that showed both a BRCA1 mutation and BRCA1 promoter methylation. This suggests that BRCA1 is mutually exclusive inactivated either by a genetic or an epigenetic event. This phenomenon was also described in the cancer genome atlas project, both for ovarian and for breast cancer (Cancer Genome Atlas Research Network, 2011; Cancer Genome Atlas Network, 2012). In Lynch's syndrome, a form of hereditary colorectal cancer, MLH1 promoter methylation has been evaluated for the selection of patients that will not be tested for germline mutations (Bettstetter et al, 2007). Recently, it has been proposed as a cost-effective pre-screening selection tool (Gausachs et al, 2012). For hereditary breast cancer screening, a similar approach could be envisaged. BRCA1 promoter methylation analysis could be used as a quick and reliable method to exclude familial-BRCA1 involvement in breast cancer patients. This is particularly important, as it has been proposed that all TNBCs below 50 years should be screened for BRCA1 and 2 mutations (Kwon et al, 2010; Robertson et al, 2012). If BRCA1 promoter methylation analysis is performed as a pre-screening assay, then a mutation can be excluded in many patients. In this study, $27-37 \%$ of all tumours showed BRCA1 promoter methylation.

A limitation is that the three series have specific selection criteria, and thus, data are not representative for the general TNBC population. The neoadjuvant series are patients treated with neoadjuvant chemotherapy and are therefore all locally advanced breast tumours. The familial-breast cancer series was a set of patients that was referred to additional molecular testing after a negative BRCA mutation test. These two groups of patients are in general younger and have more aggressive tumours than the total breast cancer population. The adjuvant series is probably the most representative of the TNBC population, as it is a consecutive series of all TNBCs treated in a community-based hospital. However, BRCAness frequencies were similar in the three series.

In conclusion, we show here that the majority of TNBCs show BRCAness, defined as a genomic BRCA1-like aCGH pattern and/ or BRCA1 promoter methylation. We show that BRCAness patients share clinical characteristics with BRCA1-mutation carriers. Finally, BRCA1-germline mutation carriers do not show BRCA1 promoter methylation in their TNBC tumours. Results should be validated in independent cohorts, and can have consequences for both screening and the treatment of this specific breast cancer subtype. 


\section{ACKNOWLEDGEMENTS}

This study was carried out within the framework of CTMM, the Center for Translational Molecular Medicine (www.ctmm.nl), project Breast CARE (030-104).

\section{CONFLICT OF INTEREST}

The authors declare no conflict of interest.

\section{REFERENCES}

Bauer KR, Brown M, Cress RD, Parise CA, Caggiano V (2007) Descriptive analysis of estrogen receptor (ER)-negative, progesterone receptor (PR)negative, and HER2-negative invasive breast cancer, the so-called triplenegative phenotype: a population-based study from the California cancer Registry. Cancer 109: 1721-1728.

Bettstetter M, Dechant S, Ruemmele P, Grabowski M, Keller G, HolinskiFeder E, Hartmann A, Hofstaedter F, Dietmaier W (2007) Distinction of hereditary nonpolyposis colorectal cancer and sporadic microsatelliteunstable colorectal cancer through quantification of MLH1 methylation by real-time PCR. Clin Cancer Res 13: 3221-3228.

Blows FM, Driver KE, Schmidt MK, Broeks A, van Leeuwen FE, Wesseling J, Cheang MC, Gelmon K, Nielsen TO, Blomqvist C, Heikkila P, Heikkinen T, Nevanlinna H, Akslen LA, Begin LR, Foulkes WD, Couch FJ, Wang X, Cafourek V, Olson JE, Baglietto L, Giles GG, Severi G, McLean CA, Southey MC, Rakha E, Green AR, Ellis IO, Sherman ME, Lissowska J, Anderson WF, Cox A, Cross SS, Reed MW, Provenzano E, Dawson SJ, Dunning AM, Humphreys M, Easton DF, Garcia-Closas M, Caldas C, Pharoah PD, Huntsman D (2010) Subtyping of breast cancer by immunohistochemistry to investigate a relationship between subtype and short and long term survival: a collaborative analysis of data for 10,159 cases from 12 studies. PLoS Med 7: e1000279.

Cancer Genome Atlas Network (2012) Comprehensive molecular portraits of human breast tumours. Nature 490: 61-70.

Cancer Genome Atlas Research Network (2011) Integrated genomic analyses of ovarian carcinoma. Nature 474: 609-615.

Fong PC, Boss DS, Yap TA, Tutt A, Wu P, Mergui-Roelvink M, Mortimer P, Swaisland H, Lau A, O'Connor MJ, Ashworth A, Carmichael J, Kaye SB Schellens JH, de Bono JS (2009) Inhibition of poly(ADP-ribose) polymerase in tumors from BRCA mutation carriers. $N$ Engl J Med 361: 123-134.

Foulkes WD, Metcalfe K, Hanna W, Lynch HT, Ghadirian P, Tung N, Olopade O, Weber B, McLennan J, Olivotto IA, Sun P, Chappuis PO, Begin LR, Brunet JS, Narod SA (2003) Disruption of the expected positive correlation between breast tumor size and lymph node status in BRCA1related breast carcinoma. Cancer 98: 1569-1577.

Gausachs M, Mur P, Corral J, Pineda M, Gonzalez S, Benito L, Menendez M, Espinas JA, Brunet J, Iniesta MD, Gruber SB, Lazaro C, Blanco I, Capella G (2012) MLH1 promoter hypermethylation in the analytical algorithm of Lynch syndrome: a cost-effectiveness study. Eur J Hum Genet 20: 762-768.

Joosse SA, Brandwijk KI, Devilee P, Wesseling J, Hogervorst FB, Verhoef S, Nederlof PM (2012) Prediction of BRCA2-association in hereditary breast carcinomas using array-CGH. Breast Cancer Res Treat 132: 379-389.

Joosse SA, Brandwijk KI, Mulder L, Wesseling J, Hannemann J, Nederlof PM (2011) Genomic signature of BRCA1 deficiency in sporadic basal-like breast tumors. Genes Chromosomes Cancer 50: 71-81.

Joosse SA, van Beers EH, Nederlof PM (2007) Automated array-CGH optimized for archival formalin-fixed, paraffin-embedded tumor material. BMC Cancer 7: 43 .

Joosse SA, van Beers EH, Tielen IH, Horlings H, Peterse JL, Hoogerbrugge N, Ligtenberg MJ, Wessels LF, Axwijk P, Verhoef S, Hogervorst FB, Nederlof PM (2009) Prediction of BRCA1-association in hereditary non-BRCA1/2 breast carcinomas with array-CGH. Breast Cancer Res Treat 116: 479-489.
Kriege M, Jager A, Hooning MJ, Huijskens E, Blom J, van Deurzen CH, Bontenbal M, Collee JM, Menke-Pluijmers MB, Martens JW, Seynaeve C (2012) The efficacy of taxane chemotherapy for metastatic breast cancer in BRCA1 and BRCA2 mutation carriers. Cancer 118: 899-907.

Kriege M, Seynaeve C, Meijers-Heijboer H, Collee JM, Menke-Pluymers MB, Bartels CC, Tilanus-Linthorst MM, Blom J, Huijskens E, Jager A, van den Ouweland A, van Geel B, Hooning MJ, Brekelmans CT, Klijn JG (2009) Sensitivity to first-line chemotherapy for metastatic breast cancer in BRCA1 and BRCA2 mutation carriers. J Clin Oncol 27: 3764-3771.

Kwon JS, Gutierrez-Barrera AM, Young D, Sun CC, Daniels MS, Lu KH, Arun B (2010) Expanding the criteria for BRCA mutation testing in breast cancer survivors. J Clin Oncol 28: 4214-4220.

Lakhani SR, van de Vijver MJ, Jacquemier J, Anderson TJ, Osin PP, McGuffog L, Easton DF (2002) The pathology of familial breast cancer: predictive value of immunohistochemical markers estrogen receptor, progesterone receptor, HER-2, and p53 in patients with mutations in BRCA1 and BRCA2. J Clin Oncol 20: 2310-2318.

Lips EH, Laddach N, Savola SP, Vollebergh MA, Oonk AM, Imholz AL, Wessels LF, Wesseling J, Nederlof PM, Rodenhuis S (2011a) Quantitative copy number analysis by Multiplex Ligation-dependent Probe Amplification (MLPA) of BRCA1-associated breast cancer regions identifies BRCAness. Breast Cancer Res 13: R107.

Lips EH, Mulder L, Hannemann J, Laddach N, Vrancken Peeters MT, van de Vijver MJ, Wesseling J, Nederlof PM, Rodenhuis S (2011b) Indicators of homologous recombination deficiency in breast cancer and association with response to neoadjuvant chemotherapy. Ann Oncol 22: 870-876.

Oonk AM, van Rijn C, Smits MM, Mulder L, Laddach N, Savola SP, Wesseling J, Rodenhuis S, Imholz AL, Lips EH (2012) Clinical correlates of 'BRCAness' in triple-negative breast cancer of patients receiving adjuvant chemotherapy. Ann Oncol 23: 2301-2305.

Robertson L, Hanson H, Seal S, Warren-Perry M, Hughes D, Howell I, Turnbull C, Houlston R, Shanley S, Butler S, Evans DG, Ross G, Eccles D, Tutt A, Rahman N (2012) BRCA1 testing should be offered to individuals with triple-negative breast cancer diagnosed below 50 years. $\mathrm{Br} J$ Cancer 106: $1234-1238$

Rodenhuis S, Mandjes IA, Wesseling J, van de Vijver MJ, Peeters MJ, Sonke GS, Linn SC (2010) A simple system for grading the response of breast cancer to neoadjuvant chemotherapy. Ann Oncol 21: 481-487.

Silver DP, Richardson AL, Eklund AC, Wang ZC, Szallasi Z, Li Q, Juul N, Leong CO, Calogrias D, Buraimoh A, Fatima A, Gelman RS, Ryan PD, Tung NM, De Nicolo A, Ganesan S, Miron A, Colin C, Sgroi DC, Ellisen LW, Winer EP, Garber JE (2010) Efficacy of neoadjuvant Cisplatin in triple-negative breast cancer. J Clin Oncol 28: 1145-1153.

Turner N, Tutt A, Ashworth A (2004) Hallmarks of 'BRCAness' in sporadic cancers. Nat Rev Cancer 4: 814-819.

Turner NC, Reis-Filho JS, Russell AM, Springall RJ, Ryder K, Steele D, Savage K, Gillett CE, Schmitt FC, Ashworth A, Tutt AN (2007) BRCA1 dysfunction in sporadic basal-like breast cancer. Oncogene 26: 2126-2132.

van der Hout AH, van den Ouweland AM, van der Luijt RB, Gille HJ, Bodmer D, Bruggenwirth H, Mulder IM, van der Vlies P, Elfferich P, Huisman MT, ten Berge AM, Kromosoeto J, Jansen RP, van Zon PH, Vriesman T, Arts N, Lange MB, Oosterwijk JC, Meijers-Heijboer H, Ausems MG, Hoogerbrugge N, Verhoef S, Halley DJ, Vos YJ, Hogervorst F, Ligtenberg M, Hofstra RM (2006) A DGGE system for comprehensive mutation screening of BRCA1 and BRCA2: application in a Dutch cancer clinic setting. Hum Mutat 27: 654-666.

Vollebergh MA, Lips EH, Nederlof PM, Wessels LF, Schmidt MK, van Beers EH, Cornelissen S, Holtkamp M, Froklage FE, de Vries EG, Schrama JG, Wesseling J, van de Vijver MJ, van Tinteren H, de Bruin M, Hauptmann M, Rodenhuis S, Linn SC (2011) An aCGH classifier derived from BRCA1mutated breast cancer and benefit of high-dose platinum-based chemotherapy in HER2-negative breast cancer patients. Ann Oncol 22: 1561-1570.

This work is published under the standard license to publish agreement. After 12 months the work will become freely available and the license terms will switch to a Creative Commons AttributionNonCommercial-Share Alike 3.0 Unported License. 